\title{
Salvage radiotherapy after radical prostatectomy: Preserving patient quality of life with modern treatment techniques
}

Martin Korzeniowski

Queen’s University, Kingston, ON, Canada

Cite as: Can Urol Assoc J 2017 Dec. 14; Epub ahead of print. http://dx.doi.org/10.5489/cuaj.5112

Published online December 14, 2017

$* * *$

In this issue of $C U A J$, readers will come across an article that showcases oncologic and functional outcomes after salvage radiation for biochemical failure for prostate cancer treated with robot-assisted radical prostatectomy (RARP). The article is particularly interesting because it involves a cohort of men treated with modern radiotherapy and surgical techniques. The disciplines of Urologic and Radiation oncology have each benefited from technological innovations and this has contributed to the improved functional outcomes seen in this study. Advances in surgical technique with the advent of RARP have shortened hospital stays, decreased perioperative complications, and improved urinary function. Similarly, radiation oncology has seen improvements in treatment planning and delivery that allow highly conformal treatments to be delivered with reduced genitourinary and gastrointestinal toxicity. ${ }^{1}$

In the paper "Functional and Oncological Outcomes of Salvage External Beam Radiotherapy following Robot-Assisted Radical Prostatectomy in a Canadian Cohort,” Ajib et al. report on the clinical outcomes for salvage radiotherapy after robot-assisted laparoscopic prostatectomy from a tertiary academic centre in Canada. A prospectively collected database included clinically relevant outcomes for urinary and sexual function. Validated measures such as the International Prostate Symptom Score (IPSS) and Sexual Health Inventory for Men (SHIM) as well as continence pad usage were used to demonstrate the safety and tolerability of salvage radiotherapy following RARP from a patient-reported outcome perspective.

While follow-up is limited, the early oncological outcomes are encouraging with good levels of PSA control despite a mean PSA of $0.5 \mathrm{ng} / \mathrm{ml}$ at the time of treatment. Men with extracapsular extension, or positive surgical margins, had excellent biochemical control $(\mathrm{PSA}<0.2)$ at 24 months (80\%, 81\% respectively) while those with seminal vesicle invasion did somewhat less well as expected (55\%). Despite the encouraging PSA results, some urologists may still be reluctant to refer patients for salvage owing to the variable clinical course after biochemical failure. Some patients have true local failure (especially those with high-risk features) and benefit from salvage treatment, while others have occult metastatic disease and 
derive greater benefit from treatment with androgen deprivation therapy. To complicate matters further, both clinicians and patients have concerns that any degree of urinary incontinence after surgery may worsen after treatment.

In the current series, Ajib et. al demonstrate that it is safe to treat patients with salvage radiation after robotic prostatectomy: men experience excellent functional urinary outcomes in addition to promising PSA control. They report that urinary quality of life is effectively unchanged from baseline (mean IPSS= 3.3) up to 24 months of follow-up (mean IPSS = 3.6). Furthermore, complete urinary continence (defined as no pads) is maintained in the majority of men at 24 months (70\%, compared to baseline 78\%, $\mathrm{p}=\mathrm{NS}$ ). Any incontinence was typically very mild, usually only requiring one pad per day. In older series, where men were treated with open radical prostatectomy and 4-field radiotherapy, the rate of complete continence (Grade 0 ) was less than $40 \%$ after treatment. ${ }^{2}$ Allowing for differences in patient selection and treatment planning volumes, Ajib et al. provide us with indirect evidence that advances in both surgical technique and radiation delivery may be contributing to the meaningful improvements in functional outcomes we see in this modern cohort of patients.

In radiation oncology we have long known that toxicity is related to volume of normal tissue irradiated. We have always been conscientious about normal tissue sparing and have exercised all options available to minimize toxicity. With advances in diagnostic imaging, treatment planning, delivery, and image-guidance, highly-conformal treatment can be administered with a very high degree of precision. This has translated into meaningful reduction in the volume of normal tissue irradiated, especially in the bladder and rectum. Considering these technological advances and their impact on reducing acute toxicity, it really comes as no surprise that we see so little change in functional urinary outcomes after salvage radiation in the current series. When offered after RARP, a large proportion of men are salvaged with radiotherapy and experience minimal change in urinary function and bother

The timing of radiation treatment after biochemical failure is also a complex decision. Large studies have not yet demonstrated a clear benefit for adjuvant over early salvage treatment with radiation ${ }^{4-6}$. However, clinical evidence consistently suggests that early treatment with lower PSAs $(\leq 0.2 \mathrm{ng} / \mathrm{ml})$ at the time of salvage radiotherapy is associated with improved treatment outcomes. Questions about the utility of adjuvant treatment remain. Does early salvage at low PSAs $(<0.2 \mathrm{ng} / \mathrm{m})$ provide the same benefits as adjuvant treatment? While we await the results of the National Cancer Institute of Canada's (NCIC CTG)Radiotherapy and Androgen Deprivation in Combination After Local Surgery trial (RADICALS, IISRCTN\#40814031), we are reassured to know that men can be safely treated with salvage radiation after RARP with minimal impact on urinary function and continence. There remains little to be gained by delaying salvage and potentially a great deal to lose. 


\section{References}

1. Michalski JM, Yan Y, Watkins-Bruner D, Bosch W, Winter K, Galvin JM, et al. Preliminary Toxicity Analysis of 3DCRT versus IMRT on the High Dose Arm of the RTOG 0126 Prostate Cancer Trial. Int J Radiat Oncol Biol Phys 2013

2. 0/08;87(5):10.1016/j.ijrobp.2013.07.041. Pearse M, Choo R, Danjoux C, Gardner S, Morton G, Szumacher E, et al. Prospective assessment of gastrointestinal and genitourinary toxicity of salvage radiotherapy for patients with prostate-specific antigen relapse or local recurrence after radical prostatectomy. Int J Radiat Oncol Biol Phys 2008 Nov 1;72(3):792-798.

3. Tendulkar RD, Agrawal S, Gao T, Efstathiou JA, Pisansky TM, Michalski JM, et al. Contemporary Update of a Multi-Institutional Predictive Nomogram for Salvage Radiotherapy After Radical Prostatectomy. JCO 2016 10/20; 2017/12;34(30):3648-3654.

4. Tendulkar RD, Agrawal S, Gao T, Efstathiou JA, Pisansky TM, Michalski JM, et al. Contemporary Update of a Multi-Institutional Predictive Nomogram for Salvage Radiotherapy After Radical Prostatectomy. JCO 2016 10/20; 2017/12;34(30):3648-3654.

5. Thompson IM, Tangen CM, Paradelo J, Lucia MS, Miller G, Troyer D, et al. Adjuvant Radiotherapy for Pathological T3N0M0 Prostate Cancer Significantly Reduces Risk of Metastases and Improves Survival: Long-Term Followup of a Randomized Clinical Trial. J Urol 2017/12;181(3):956-962.

6. Wiegel T, Bottke D, Willich N, Semjonov A, Siegmann A, Stoeckle MC, et al. Phase III Results of Adjuvant Radiotherapy Versus”Wait and See”in Patients With pT3 Prostate Cancer Following Radical Prostatectomy (ARO 96-02/AUO AP 09/95). ; 2007. 